\title{
Surgical Navigation Recording Systems in Orbitozygomatic Traumatology
}

\author{
Giorgio Novelli, MD, Gabriele Tonellini, MD, Fabio Mazzoleni, MD, Davide Sozzi, MD, \\ and Alberto Bozzetti, MD
}

\begin{abstract}
An essential step in the navigation procedure is the recording of markers required for the triangulation of the intraoperative navigation system. The aim of this study was to describe the procedure and preliminary results of a simple methodology for the application of dental markers to achieve good triangulation of the navigation system in maxillofacial surgery, highlighting the indications, contraindications, and possible limitations.

We analyzed results from a sample of 7 patients with orbital or orbitozygomatic fractures, who were subjected to surgical intervention for the reduction and synthesis of the zygomatic fracture and from 1 case of untreated orbital fracture with enophthalmos and diplopia. We used 2 different types of dental markers: in 3 patients, we used 4 or 5 ordinary orthodontic brackets, which we placed on their upper maxillary teeth, and in 1 patient, the hexagonal-headed screws used in osteosynthesis. The accuracy of the recording was assessed during surgery by checking healthy anatomic structures against computed tomographic images. By analyzing our clinical results in light of the most recent literature, we highlighted that the application of dental markers is comparable with the best recording systems requiring a discrepancy of less than $1 \mathrm{~mm}$.

From the preliminary clinical analysis of the results, we confirm that the introduction of this new and simple procedure enables the successful triangulation of the navigation system, which can be used whenever the use of a navigator is required.
\end{abstract}

Key Words: Surgical navigation, orbitozygomatic traumatology, dental markers

(J Craniofac Surg 2012;23: 890-892)

E irst introduced in oral and maxillofacial surgery in the early F 1990s, surgical navigation has gradually played a bigger role in medical practice in maxillofacial surgery units. The three-dimensional complexity of facial anatomy requires accurate three-dimensional reconstruction to ensure a good aesthetic result as well as functional recovery. At present, computer-assisted maxillofacial surgery is mostly used in traumatology. However, its use is becoming more common in

From the Department of Maxillofacial Surgery, University of MilanoBicocca, San Gerardo Hospital, Monza, Italy.

Received September 4, 2011.

Accepted for publication January 2, 2012.

Address correspondence and reprint requests to Giorgio Novelli, MD, Department of Maxillofacial Surgery, San Gerardo Hospital Via Pergolesi, 33, Monza (MB), 20900 Italy; E-mail: g.novelli@hsgerardo.org; nove.gio@gmail.com

The authors report no conflicts of interest.

Copyright $($ C) 2012 by Mutaz B. Habal, MD

ISSN: $1049-2275$

DOI: 10.1097/SCS.0b013e31824e6993 the field of oncology (resection control and reconstruction), in craniofacial surgery for severe malformations, and in orthognathic surgery. ${ }^{1-4}$

An essential step in the navigation procedure is the recording of markers required for the triangulation of the intraoperative navigation system with the virtual plan that the surgeon defines on the computed tomography (CT) through the information processing software. ${ }^{2,5-7}$ A mistake at this stage could affect the accuracy of the surgical procedure. ${ }^{8}$

Successful triangulation requires at least 4 markers, appropriately distanced one from the other. ${ }^{2,6}$ It is essential that the marker positioned before the $\mathrm{CT}$ is absolutely stable and easy to access, as it is subsequently used throughout surgery, both during the first recording session and during any future recordings required.

There are 2 recording methods: invasive and noninvasive. ${ }^{2,5-7,9,10}$ Invasive procedures require the use of intraoral or extraoral bone markers. Minimally invasive surgical incisions under local anesthesia or the presence of trauma-induced wounds are needed for the purposes of the procedure. ${ }^{4,7}$

The main advantage of these procedures is the absolute stability of the marker, which is positioned on rigid bones that are not affected by the surgical operation. The disadvantages consist in needing a small surgical intervention under local anesthetic and in having to leave the marker until the day of surgery; this does not prove very practical in the case of elective surgery.

Noninvasive procedures use anatomic landmarks such as prominent bones, which are first identified on the CT and then used to triangulate the navigation system. Alternatively, skin adhesives are used; these have the advantage of being easy to apply but have poor accuracy levels and may be conditioned by the presence of soft tissue edema. Moreover, various kinds of resin-based tooth splints with radiopaque markers are described. ${ }^{2,3,6,7}$

Ultimately, laser surface scanning systems may also be used, but these require wide exposure of bone surfaces, or in skin scanning, they may be affected by the surgical incision, which prevents the triangulation from being repeated, if necessary. ${ }^{2,6,9,11}$

The aim of this study was to describe the procedure and preliminary results of a simple methodology for the application of dental markers to achieve good triangulation of the navigation system in maxillofacial surgery, highlighting the indications, contraindications, and possible limitations.

\section{MATERIALS AND METHODS}

We analyzed results from a sample of 7 patients with orbital or orbitozygomatic fractures, who were subjected to surgical intervention for the reduction and synthesis of the zygomatic fracture and from 1 case of untreated orbital fracture with enophthalmos and diplopia (Table 1).

As inclusion criterion, we selected patients with unilateral orbital and/or zygomatic fractures with no lesions to the upper maxillary and with a sufficient number of permanent teeth (at least 5).

For recording purposes, we used dental markers in all patients, which we positioned before performing the $\mathrm{CT}$ of their facial structure. 
TABLE 1. Clinical Case Summaries

\section{Blow-out fracture}

Orbitozygomatic fractures

Untreated orbital fracture

We used 2 different types of dental markers: in 3 patients, we used 4 or 5 ordinary orthodontic brackets, which we placed on their upper maxillary teeth (Fig. 1), and in 1 patient, the hexagonal-headed screws used in osteosynthesis attached with dental composite resins (Fig. 2).

As programming software, we used the virtual 3D iPlan 2.6 and 3.0 Brainlab, and as navigation system, Brainlab VectorVision (Brainlab, Munich, Germany). The virtual plan was done by mirroring the healthy side onto the fractured side.

The recording process involves defining reference landmarks on a preoperative CT scan within the planning software. These landmarks assume a specific spatial position in the three-dimensional reconstruction of CT data.

Subsequently, CT data need to be located within the depth of the operation site before the intervention takes place, so that they match the patient's actual position within the surgical area. This procedure is known as "recording."

Localization takes place, thanks to optical references, which allow the continuous tracking of points in space. They consist of reflective spheres that the navigator identifies through infrared video cameras. Reference systems mounted must be as "stable" as possible and should not move in relation to the landmarks. Landmarks, which were defined during the planning stage and of which we know all the relative reciprocal distances in space, are identified one by one in the operation site. The software is able to process their $\mathrm{CT}$ theoretical positioning and to determine their real location on the patient. Once the landmarks have been located, all CT/magnetic resonance data sets introduced within the operating surface are also positioned virtually.

For intraoperative recording, we used a star-shaped tool, which was fixed directly onto the skull to ease its movement during the surgical operation (Figs. 3A, B). The accuracy of the recording was assessed during surgery by checking healthy anatomic structures against CT images. It was also assessed after surgery by comparing preoperative $\mathrm{CT}$ and the relevant mirroring with the postoperative $\mathrm{CT}$ and by measuring the validity of the result (Fig. 4).

The analysis of preoperative and postoperative images was conducted with the same software used for surgical navigation (iPlan 2.6/3.0 CMF-Brainlab) by merging preoperative and postoperative images and comparing the reduction and/or reconstruction quality.

\section{RESULTS}

Clinical and radiologic data were analyzed, to evaluate both the accuracy of the recording and the clinical result. In all the cases treated, no discrepancies were found between the patient's anatomy and the CT images. During the recording process, discrepancies less than or equal to $1 \mathrm{~mm}$ were observed, as described in Table 2 . We

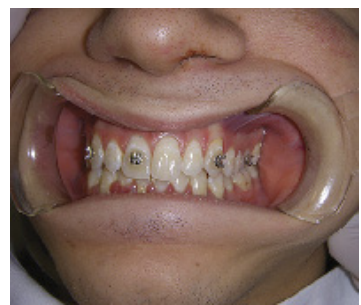

FIGURE 1. Orthodontic brackets.

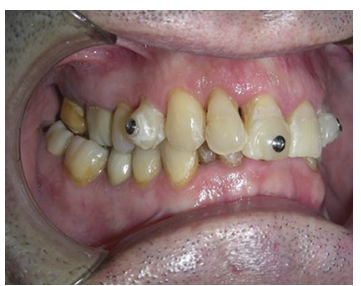

FIGURE 2. Hexagonal-headed screws.
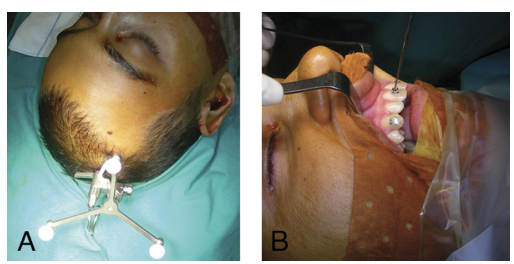

FIGURE 3. A and B, Star-shaped tool and intraoperative registration points.

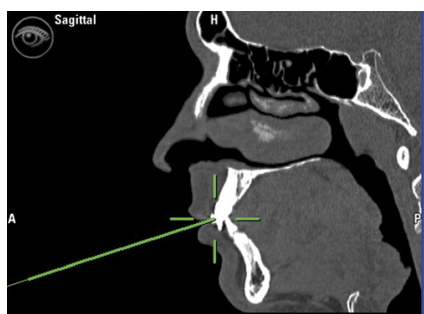

FIGURE 4. Intraoperative screenshot from navigation system showing a correct registration.

\begin{tabular}{|c|c|c|c|}
\hline & Type of Fracture & Accuracy, mm & Registration Technique \\
\hline 1 & Blow-out fracture & $<1$ & Hexagonal-headed screws \\
\hline 2 & Blow-out fracture & $<1$ & Brackets \\
\hline 3 & Orbitozygomatic fractures & $<1$ & Brackets \\
\hline 4 & Orbitozygomatic fractures & $\leq 1$ & Brackets \\
\hline 5 & Orbitozygomatic fractures & $<1$ & Brackets \\
\hline 6 & Untreated orbital fracture & $<1$ & Brackets \\
\hline 7 & Orbitozygomatic fractures & $\leq 1$ & Hexagonal-headed screws \\
\hline
\end{tabular}


TABLE 3. Protocol Currently Followed in the Authors' Department

\begin{tabular}{|c|c|c|}
\hline Type of Fracture & Clinical Status & Registration Technique \\
\hline Blow-out fracture, orbitozygomatic fractures & $\begin{array}{c}\text { Procedure on orbital cavities and midface } \\
\text { Acute trauma } \\
\text { Untreated orbital fracture }\end{array}$ & $\begin{array}{l}\text { Orthodontic brackets } \\
\text { Hexagonal-headed screws } \\
\text { Occlusal splint alone }\end{array}$ \\
\hline $\begin{array}{l}\text { Fractures located above the orbital plane with no lesions to the upper } \\
\text { maxillary and with a sufficient number of permanent teeth }\end{array}$ & $\begin{array}{l}\text { Acute trauma } \\
\text { Untreated craniofacial fractures }\end{array}$ & $\begin{array}{l}\text { Orthodontic brackets or hexagonal-headed screws }+ \text { bone } \\
\text { implant markers on lateral orbitae }\end{array}$ \\
\hline $\begin{array}{l}\text { Blow-out fracture, orbitozygomatic fractures in edentulous patients } \\
\text { or those with periodontal disease }\end{array}$ & $\begin{array}{l}\text { Procedure on orbital cavities and midface } \\
\text { Acute trauma }\end{array}$ & Bone implant markers on lateral orbitae \\
\hline $\begin{array}{l}\text { Fractures located above the orbital plane (craniofacial trauma) } \\
\text { in edentulous patients or those with periodontal disease }\end{array}$ & $\begin{array}{l}\text { Acute trauma } \\
\text { Untreated craniofacial fractures }\end{array}$ & Bone implant markers \\
\hline
\end{tabular}

did not notice significant clinical differences between the recording carried out with titanium screws and orthodontic brackets.

Postoperative CT follow-up, analyzed through image fusion, confirmed the results of the surgical navigation and the successful reduction and/or reconstruction in terms of positioning, which was programmed based on mirroring and on the anatomic profile of bone structures.

From the clinical assessment, we found that the results obtained in terms of facial symmetry, exophthalmos correction, and diplopia resolution were good. By analyzing our results in light of the most recent literature, ${ }^{6,7}$ we highlighted that the application of dental markers is comparable with the best recording systems requiring a clinical discrepancy of less than $1 \mathrm{~mm}$.

\section{DISCUSSION}

Surgical navigation provides us with the opportunity to reconstruct bone structures to the best of our ability, allowing the operator to check the virtual plan and the surgical outcome during the surgical procedure. Moreover, it allows the surgeons to find direction through various anatomic structures and to verify and correct the reconstruction they already carried out. A crucial step in surgical navigation is the recording of markers. A mistake at this stage can cause the surgeon to make a surgical error, thus resulting in a poor result. ${ }^{5}$

As shown in the literature, the best recording systems in the craniomaxillofacial field are those involving fixed registration points. ${ }^{2-4,6,7}$ The above methods include both invasive (bone screws) and noninvasive procedures (tooth splints) and have an error of less than or equal to $1 \mathrm{~mm}$. Compared with surface registration procedures (laser scan and skin adhesive markers), they allow to repeat the registration process during surgery if the reference systems become mobile, and the navigation signal is lost. Moreover, surface registration procedures are restricted as they cannot be used in cases of soft tissue edema because the error can exceed 4 to $5 \mathrm{~mm}$.

The protocol that we usually follow requires the use of dental markers (orthodontic brackets or the head of screws used in osteosynthesis) in cases where the acquisition of CT images shortly precedes the execution of the surgical intervention. This procedure offers the advantages of being easy, cheap, and fast to implement especially in the field of traumatology. Its limitation is that it requires an adequate number of permanent teeth on the maxillary arch, which have to be stable. If these conditions are not met, we use adequately distributed bone-anchoring screws, avoiding skin incisions if possible and exploiting traumatic wounds, if there are any.

We use occlusal splints after fractures where the acquisition of CT images takes place at a different time than the surgical intervention. Thanks to this, the patients are not required to wear markers (bone screws or dental references) while waiting for their surgical intervention. Even in this case, its limitations are the presence of teeth, the stability of the maxilla, and the difficult implementation in emergency traumatology. Moreover, in cases where it is necessary to check anatomic areas located above the orbital surface, this procedure can be easily integrated with surface scanning systems or fixed registration systems (bone-anchoring screws), to increase the accuracy of triangulation. ${ }^{6}$ The protocol that is currently followed in our department is summarized in Table 3.

\section{CONCLUSIONS}

From the preliminary analysis of the results, we confirm that the introduction of this new and simple procedure enables the successful triangulation of the navigation system, which can be used whenever the use of a navigator is required. Extending the application of this procedure to different scenarios both within traumatology and other pathologies will enable us to validate the experience in orbitozygomatic traumatology.

\section{REFERENCES}

1. Hassfeld S, Mühling J, Zöller J. Intraoperative navigation in oral and maxillofacial surgery. Int J Oral Maxillofac Surg 1995;24:11-119

2. Schramm A, Gellrich NC, Schmelzeisen R. Navigational Surgery of the Facial Skeleton. Berlin, Germany: Springer, 2007

3. Schramm A, Suarez-Cunqueiro MM, Rücker M, et al. Computer-assisted therapy in orbital and mid-facial reconstructions. Int J Med Robot 2009;5:111-124

4. Yu H, Shen G, Wang X, et al. Navigation-guided reduction and orbital floor reconstruction in the treatment of zygomatic-orbital-maxillary complex fractures. J Oral Maxillofac Surg 2010;68:28-34

5. Eggers G, Mühling J, Marmulla R. Image-to-patient registration techniques in head surgery. Int J Oral Maxillofac Surg 2006;35: 1081-1095

6. Lüebbers Ht, Messmer P, Obwegeser JA, et al. Comparison of different registration methods for surgical navigation in cranio-maxillofacial surgery. J Craniomaxillofac Surg 2008;36:109-116

7. Lübbers H-T, Matthews F, Zemann W, et al. Registration for computer-navigated surgery in edentulous patients: a problem-based decision concept. J Craniomaxillofac Surg 2011;39:453-458

8. Widmann G, Stoffner R, Bale R. Errors and error management in image-guided craniomaxillofacial surgery. Oral Surg Oral Med Oral Pathol Oral Radiol Endod 2009;107:701-715

9. Marmulla R, Mühling J, Lüth T, et al. Advanced surface-recording techniques for computer-assisted oral and maxillofacial surgery. Br J Oral Maxillofac Surg 2004;42:511-519

10. Bradley Strong E, Rafii A, Holhweg-Majert B, et al. Comparison of 3 optical navigation systems for computer-aided maxillofacial surgery. Arch Otolaryngol Head Neck Surg 2008;134:1080-1084

11. Marmulla R, Lüth T, Mühling J, et al. Automated laser registration in image-guided surgery: evaluation of the correlation between laser scan resolution and navigation accuracy. Int J Oral Maxillofac Surg 2004:33:642-648 